\title{
La biosynthèse des acides aminés à chaîne branchée et des purines : deux voies essentielles pour une croissance optimale de Streptococcus thermophilus dans le lait
}

\author{
Peggy Garault ${ }^{\mathrm{a}}$, Catherine LetORT $^{\mathrm{b}}$, Vincent JUILlarD ${ }^{\mathrm{b}}$, \\ Véronique MONNET $^{\mathrm{a} *}$ \\ a Unité de Biochimie et Structure des Protéines, INRA, 78352 Jouy-en-Josas Cedex, France \\ ${ }^{\mathrm{b}}$ Unité de Recherches Laitières et Génétique Appliquée, INRA, \\ 78352 Jouy-en-Josas Cedex, France
}

\begin{abstract}
Branched-chain amino acids and purine biosynthesis: two pathways essential for optimal growth of Streptococcus thermophilus in milk. Slowing down and delays in Streptococcus thermophilus growth in milk have negative effects for dairy technology. Our objectives are to understand the reasons for those delays, and to identify some of the functions essential for optimal growth of $S$. thermophilus in milk. To reach this goal, a random insertional mutagenesis was performed on a $S$. thermophilus strain chosen for its ability to grow rapidly in milk. We obtained 14 different mutants, affected in their capacity to grow quickly in milk. Four of them were further characterized: two were mutated in the branched-chain amino acids biosynthesis operon and two others were mutated in the purine nucleotides biosynthesis operon. Consequently, branched-chain amino acids and purine biosynthesis pathways are functional in S. thermophilus. Our results suggested that the amount of both branched-chain amino acids and purine nucleotides, present in milk, and directly consumable is not sufficient for an optimal growth. Moreover, we identified the branched-chain amino acids and purine biosynthesis pathways as essential functions for optimal growth of $S$. thermophilus in milk.
\end{abstract}

milk / Streptococcus thermophilus / growth / branched chain amino acid / purine

Résumé - Les retards ou ralentissements de croissance de Streptococcus thermophilus, parfois observés dans le lait, ont des conséquences négatives importantes sur le plan technologique. Notre objectif est de comprendre les raisons de ces retards et d'identifier les fonctions qui, chez $S$. thermophilus, sont essentielles à la croissance optimale dans le lait. Pour atteindre cet objectif, une mutagenèse insertionnelle au hasard a été réalisée sur une souche de $S$. thermophilus choisie pour sa capacité à se développer rapidement dans le lait. Les mutants pour lesquels la croissance n'est plus optimale dans le lait ont été sélectionnés. Nous avons obtenu 14 mutants distincts affectés dans leur

* Correspondance et tirés-à-part

Tél. : (33) 134652149 ; fax : (33) 134652163 ; e-mail : monnet @ jouy.inra.fr 
capacité à se développer rapidement dans le lait. Ceux-ci ont été classés en trois groupes selon leur comportement dans différents milieux de culture. Quatre mutants ont été mieux caractérisés : 2 sont atteints dans l'opéron de la biosynthèse des acides aminés à chaîne branchée et les 2 autres dans l'opéron de la biosynthèse des bases puriques. Les voies de biosynthèse des acides aminés à chaîne branchée et des bases puriques sont donc fonctionnelles chez $S$. thermophilus. Nos résultats suggèrent que les quantités d'acides aminés à chaîne branchée et de bases puriques, assimilables dans le lait par $S$. thermophilus, ne suffisent pas à une croissance optimale. Ils mettent de plus en évidence que les voies de biosynthèse des acides aminés à chaîne branchée et des purines sont deux fonctions essentielles à la croissance de $S$. thermophilus dans le lait.

lait / Streptococcus thermophilus / croissance / acide aminé à chaîne ramifiée / base purique

\section{INTRODUCTION}

La principale propriété recherchée chez les bactéries lactiques, utilisées en tant que levains en fabrication fromagère, est leur capacité à acidifier le lait et à se développer de façon régulière. En effet, ce sont, sans nul doute, les irrégularités observées au niveau de leur croissance qui limitent la maîtrise des bactéries lactiques en technologie laitière. Dans le lait, elles doivent trouver un certain nombre de nutriments nécessaires à leur croissance, et, en particulier, les acides aminés pour lesquels elles sont auxotrophes $[4,5,16,17]$.

Les principales fonctions, qui, chez les bactéries lactiques ont été identifiées comme essentielles à la croissance optimale dans le lait, l'ont surtout été chez les lactocoques et concernent la nutrition azotée. Ainsi, la présence d'une protéase de paroi, d'un transporteur d'oligopeptides et de certaines peptidases intracellulaires $[15,23,26]$ sont nécessaires au développement rapide de L. lactis dans le lait. Des voies de biosynthèse comme celle de l'aspartate [27] et celle des bases puriques sont également identifiées comme nécessaires pour une croissance optimale dans le lait chez les lactocoques [18]. Pour $S$. thermophilus, qui est moins exigeante que $L$. lactis d'un point de vue nutritionnel [6], les données sur les fonctions qui sont essentielles à sa croissance optimale sont plus rares. Toutefois, une protéase de paroi conditionnerait, comme chez les lactocoques, la croissance optimale de $S$. thermophilus dans le lait [22]. Il semble, en particulier, que l'apport d'acide glutamique, d'histidine et de méthionine soit un élément limitant pour la croissance de $S$. thermophilus dans le lait [1]. Au cours de ce travail, nous avons mis en évidence l'importance de deux voies de biosynthèse, celle des acides aminés à chaîne ramifiée et celle des purines pour la croissance optimale de $S$. thermophilus dans le lait.

\section{MATÉRIEL ET MÉTHODES}

\subsection{Mutagenèse}

Une mutagenèse insertionnelle au hasard a été effectuée dans une souche de $S$. thermophilus à croissance rapide dans le lait (ST18, fournie par Rhodia-Food). La mutagenèse a été réalisée à l'aide du plasmide pG+host9::ISS1 selon la méthode décrite par Maguin et al. [14].

\subsection{Cribles et milieux de cultures}

Plus de 10.000 mutants ont été criblés en présence d'érythromycine $5 \mu \mathrm{g} \cdot \mathrm{mL}^{-1}$ sur milieu FSDA (Fast Slow Differential Agar) [12] qui permet de distinguer les colonies à croissance lente ou limitée dans le lait, de celles qui y poussent rapidement ou de façon optimale. Les mutants retenus ont ensuite 
été cultivés dans du lait NILAC (10\% m/vol) (Nilac, Nederlands Instituut von Zuivelonderzoek, Ede, Pays-Bas) autoclavé à $110^{\circ} \mathrm{C}$, 12 min, tamponné par du glycerophosphate de sodium $0.75 \mathrm{mmol} \cdot \mathrm{L}^{-1}$ et contenant, dans certains cas, de l'extrait de levure ou de la casitone $(3 \% \mathrm{~m} / \mathrm{vol})$. La croissance a été suivie par mesure de la densité optique à $480 \mathrm{~nm}$ après transparisation du lait par dilution au 1/10 e avec de l'EDTA pH 12 [24]. Certains mutants ont également été cultivés dans un milieu chimiquement défini (MCD) dans lequel tous les acides aminés étaient fournis sous forme libre [19]. Des expériences d'omission d'acides aminés à chaîne branchée ont été effectuées dans ce milieu. Les croissances à $37{ }^{\circ} \mathrm{C}$ dans ce milieu ont été suivies par mesure de la D.O. à $600 \mathrm{~nm}$ en continu dans un lecteur de microplaques Microbiology Reader Bioscreen C (Labsystems, Helsinki, Finlande). Chaque puits contenait $200 \mu \mathrm{L}$ de milieu de culture et était ensemencé à $4 \%$ (vol/vol) avec des cellules lavées et resuspendues dans du tampon phosphate $50 \mathrm{mmol} \cdot \mathrm{L}^{-1} \mathrm{pH} 7$.

\subsection{Identification des gènes interrompus}

Ils ont été clonés chez $E$. coli et identifiés par séquençage comme précédemment décrit [14]. Le kit Dye Terminator ainsi qu'un séquenceur 310 Genetic Analyzer (Applied Biosystems, Foster City, EU) ont été utilisés pour le séquençage de l'ADN. Les séquences d'ADN ont été analysées avec les logiciels Genetics Computer Group sequence analysis de l'université du Wisconsin [7] et avec Mail Fasta (National Center for Biotechnology Information).

\section{RÉSULTATS}

\subsection{Mutagenèse et obtention de trois groupes de mutants}

Parmi les 10000 intégrants, résistants à l'érythromycine, obtenus, 14 ont été retenus pour leur incapacité à se développer rapidement dans le lait. Leur croissance dans le lait additionné d'extrait de levure et dans le lait additionné de casitone nous a permis de les classer en 3 groupes distincts. Le premier groupe était constitué de 3 mutants, dont la croissance dans le lait était stimulée tant par l'addition de casitone que par l'ajout d'extrait de levure. Le deuxième groupe était constitué de 7 mutants dont la croissance dans le lait était stimulée par l'addition d'extrait de levure mais pas par l'addition de casitone. Enfin le dernier groupe était constitué de 4 mutants, dont la croissance dans le lait n'était stimulée ni par l'addition de casitone ni par celle d'extrait de levure. Cette classification nous a permis dans un premier temps de prédire le type de la fonction touchée dans chacun des mutants. Ainsi, les mutants dont la croissance dans le lait est stimulée par la casitone sont vraisemblablement affectés dans la nutrition azotée. Les mutants dont la croissance dans le lait est stimulée par l'addition d'extrait de levure et pas par l'addition de casitone ne sont probablement pas affectés dans la nutrition azotée mais dans un autre métabolisme.

\subsection{La voie de biosynthèse des purines}

Deux mutants dont la croissance dans le lait était stimulée par l'addition d'extrait de levure mais pas par l'addition de casitone (Fig. 1), étaient mutés dans la voie de biosynthèse des bases puriques (Fig. 2). Le premier était muté dans le gène purC codant la SAICAR synthétase et le deuxième dans le gène purH codant l'inositol phosphate synthétase. Ces mutants ont une croissance quasi nulle dans le lait après $8 \mathrm{~h}$ de culture. Cette voie de biosynthèse doit donc être active et est essentielle pour la croissance dans le lait de la souche de $S$. thermophilus étudiée.

L'addition d'extrait de levure ou de bases puriques (résultats non présentés) dans le 


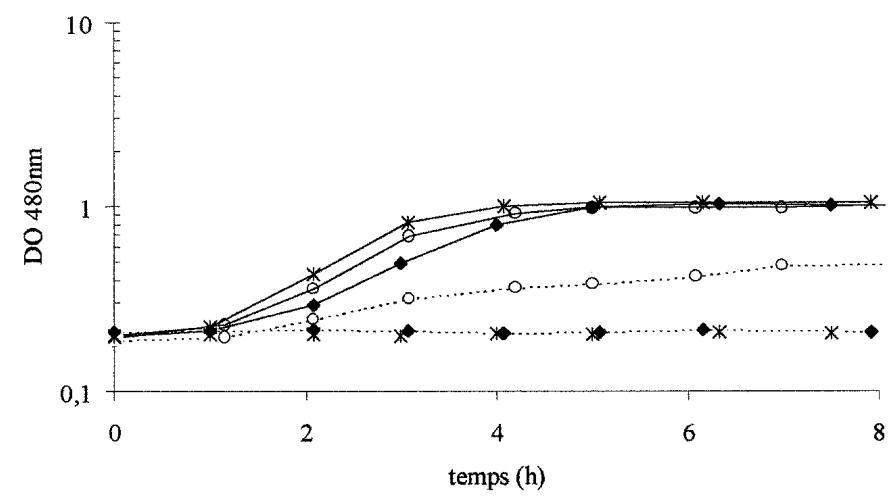

Figure 1. Courbes de croissance obtenues dans le lait $(\diamond)$, le lait additionné de casitone $(\star x)$ ou d'extrait de levure $(\bigcirc)$ avec la souche sauvage $(-)$ ou le mutant $\mathrm{PurC}^{-}(----)$.

Figure 1. Growth curves obtained in milk $(\diamond)$, in milk containing casitone $(X)$, in milk containing yeast extract $(\bigcirc)$, with the wild type strain $(-)$ or with the $\mathrm{PurC}^{-}$mutant $(---\cdot)$.

lait permet de restaurer la croissance optimale des mutants. Cela indique que le transport des bases n'est pas limitant chez la souche de $S$. thermophilus étudiée et que la quantité de bases puriques dans le lait est insuffisante pour une croissance normale de la souche.

\subsection{La voie de biosynthèse des acides aminés à chaîne ramifiée}

Deux autres mutants, dont la croissance dans le lait était stimulée par l'addition, soit

5-phosphoribosyl-pyrophosphate

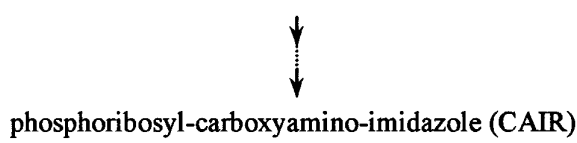

purC

phosphoribosyl-succinocarboxamide-amino-imidazole (SAICAR)

$\downarrow$

purH

IMP

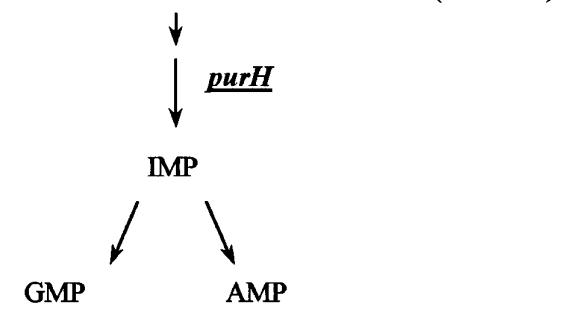

de casitone, soit d'extrait de levure (Fig. 3), étaient mutés dans les gènes de biosynthèse des acides aminés à chaîne ramifiée (AACR ; leucine, valine et isoleucine ; Fig. 4). Nous avons obtenu un mutant atteint dans le gène $i l v B$ codant l'acétolactate synthétase et un mutant atteint dans le gène ilvC codant la céto-acide réducto-isomérase.

Nous avons comparé le taux de croissance maximum, obtenu pour la souche sauvage, dans le MCD contenant les trois acides aminés à chaîne ramifiée et dans un MCD privé de ces trois acides aminés. En présence

Figure 2. Schéma de la voie de synthèse des bases puriques.

Figure 2. Schematic representation of the purines biosynthesis pathway. 


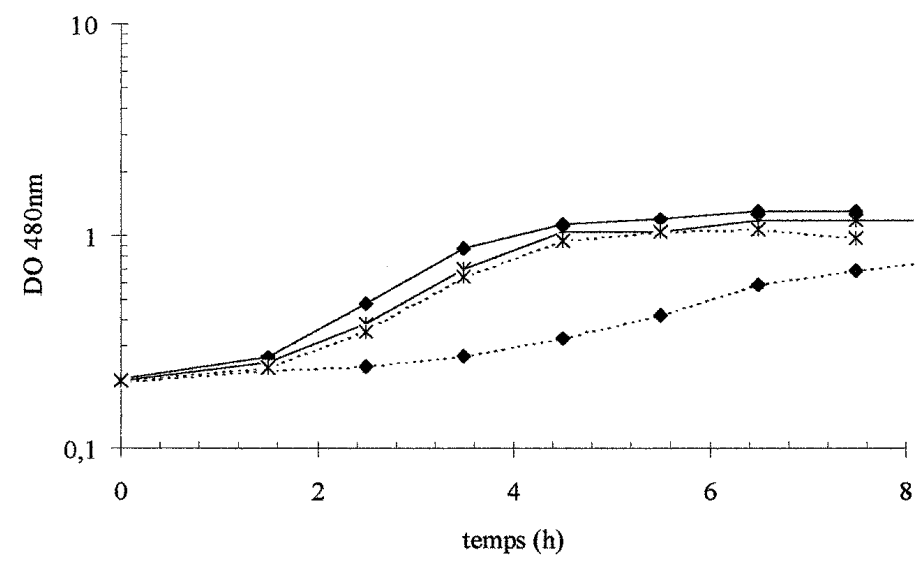

Figure 3. Courbes de croissance obtenues dans le lait $(\diamond)$, ou le lait additionné de casitone $(\star)$ avec la souche sauvage (-) ou le mutant $\mathrm{IlvC}^{-}(----)$.

Figure 3. Growth curves obtained in milk $(\diamond)$, in milk containing casitone $(\Varangle)$, with the wild type strain $(-)$ or with the $\mathrm{IlvC}^{-}$mutant (----).

des 3 acides aminés à chaîne ramifiée le taux de croissance obtenu est de $1,2 \mathrm{~h}^{-1}$ alors qu'en absence de ces 3 acides aminés il n'est que de $0,4 \mathrm{~h}^{-1}$ (Fig. 5). Pour la souche de S. thermophilus étudiée, la biosynthèse des AACR est donc active puisqu'il y a une croissance dans le MCD sans Ile, Leu et Val. Toutefois, elle est insuffisante pour obtenir une croissance optimale, car le taux de croissance diminue de deux tiers sans les 3 AACR. Des expériences complémentaires portant sur 12 autres souches industrielles de $S$. thermophilus, montrant que la voie de biosynthèse des AA,CR est aussi active, indiquent donc que ce caractère semble répandu dans cette espèce (résultats non
Figure 4. Schéma de la biosynthèse des acides aminés à chaîne ramifiée.

Figure 4. Schematic representation of the branched chain amino acids biosynthesis pathway.

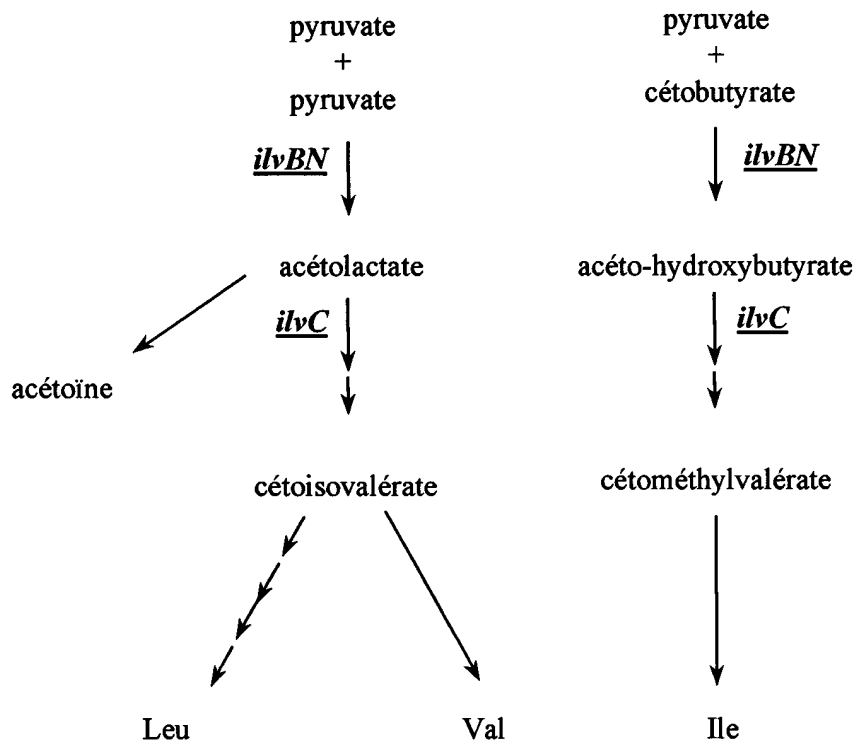




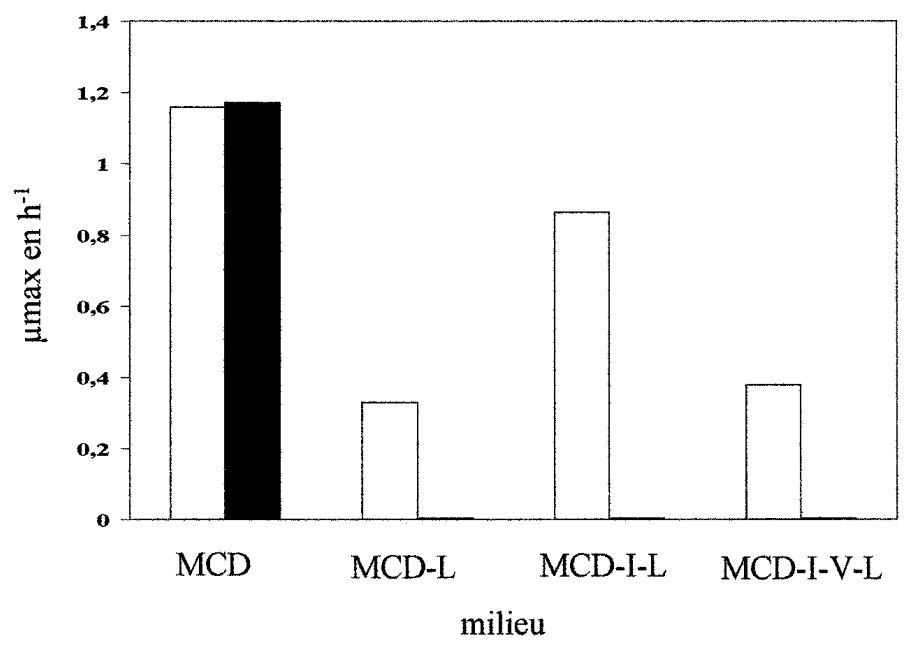

Figure 5. Taux de croissance obtenus par la souche sauvage ( $\square$ ) ou le mutant $\mathrm{IlvB}^{-}$(ou le mutant $\mathrm{IlvC}^{-}$) dans différents milieux MCD (I pour isoleucine, V pour valine et L pour leucine).

Figure 5. Growth rates of the wild type strain $(\square)$ and the $\mathrm{IlvB}^{-}$(or IlvC ${ }^{-}$) mutant $(\square)$ in different chemically defined media (I for isoleucine, $\mathrm{V}$ for valine and $\mathrm{L}$ for leucine).

présentés). La comparaison des taux de croissance de la souche sauvage dans le MCD privé de Leu et Ile avec celui dans le MCD privé seulement de Leu (Fig. 5) montre un effet inhibiteur de l'isoleucine. Les taux de croissance obtenus sont de 0,9 et $0,3 \mathrm{~h}^{-1}$ respectivement. La présence d'Ile dans le milieu semble donc réprimer la synthèse de la leucine.

En parallèle, nous avons réalisé la croissance des mutants $i l v B$ et $i l v C$ en MCD total ou sans Ile, Leu et Val. En présence des 3 AACR le taux de croissance obtenu est similaire à celui de la souche sauvage alors qu'en absence des 3 AACR, il y a absence totale de croissance.

\section{DISCUSSION}

L'obtention de clones de $S$. thermophilus dont la croissance dans le lait est affectée par une mutagenèse au hasard, nous a permis d'identifier 2 voies de biosynthèse, fonctionnelles chez cette espèce, qui conditionnent la croissance optimale dans le lait.
La nécessité de synthèse des bases puriques par $S$. thermophilus est liée à la pauvreté du lait pour ces bases. Chez L. lactis, cette même voie de biosynthèse est également nécessaire pour une croissance régulière dans le lait [18]. Le rôle des bases puriques dans cette croissance peut être direct (fourniture des bases pour les acides nucléiques) ou indirect. En effet, chez le lactocoque, les bases sont des précurseurs de régulateurs de stress, acide en particulier [20].

En ce qui concerne la voie de biosynthèse des AACR, nous avons montré qu'elle était fonctionnelle chez $S$. thermophilus comme pour les souches de L. lactis d'origine végétale, alors que ce n'est pas le cas pour les souches laitières de L. lactis $[9,10]$ ou pour Leuconostoc mesenteroides [3]. La voie de biosynthèse des AACR, quand elle est fonctionnelle, est soumise à des phénomènes de régulation à différents niveaux, et en particulier à des phénomènes de rétro-inhibition. Il semble que ce soit également le cas chez S. thermophilus (Fig. 5). Ces résultats sont à rapprocher de ceux de Braquart et al. [2] 
qui avaient observé un effet négatif de l'ajout d'isoleucine au lait sur la croissance de $S$. thermophilus.

Chez les autres bactéries, les gènes $i l v$ sont organisés en opéron. Chez la souche de $S$. thermophilus étudiée, nous avons séquencé un fragment d'ADN contenant 4 phases ouvertes de lecture ayant des homologies avec les gènes $i l v D, B, N$ et $C$ de L. lactis et semblant également être organisées en opéron [8]. De plus, nous avons mis en évidence un promoteur et un terminateur respectivement en amont et en aval du gène $i l v C$. Ce dernier gène peut être transcrit indépendamment des autres gènes ilv puisque des expériences de Northern ont permis la mise en évidence du transcrit correspondant à ce gène [8]. Cette transcription indépendante du gène $i l v C$ a déjà été observée chez d'autres bactéries, comme E. coli [28] et Corynebacterium glutamicum [13], mais pas chez les autres bactéries lactiques.

L'acétolactate, substrat de la céto-acide réducto-isomérase (IlvC) se situe à un carrefour métabolique. En effet, c'est un intermédiaire dans la synthèse de la leucine et de la valine, mais aussi dans la voie de formation de l'acétoïne. Cette transcription indépendante du gène $i l v C$, pourrait, chez $S$. thermophilus, éviter l'accumulation de l'acétolactate, potentiellement toxique. De cette façon, elle jouerait un rôle similaire à celui de l'acétolactate décarboxylase de L. lactis qui permet la formation de l'acétoïne à partir de l'acétolactate [11]. De plus, la voie de l'acétoïne joue un rôle dans le maintien du $\mathrm{pH}$ interne des bactéries en changeant les métabolites acides en composés neutres, et participe également à la régénération du $\mathrm{NAD}^{+}[21,25]$.

\section{REMERCIEMENTS}

Le travail a été financé par Danone, RhodiaFood et Sodiaal dans le cadre du contrat « Substrats de fermentation ». Les auteurs remercient A. Sepulchre, P. Ramos, J. Mengaud et F. Rul pour les nombreuses discussions fructueuses qu'ils ont eues avec eux au cours de cette étude.

\section{RÉFÉRENCES}

[1] Braquart P., Lorient D., Effet des acides aminés sur la croissance de Streptococcus thermophilus, Milchwissenschaft 32 (1977) 221-224.

[2] Braquart P., Lorient D., Alais C., Effet des acides aminés sur la croissance de Streptococcus thermophilus. II. Étude sur 5 souches, Milchwissenschaft 33 (1978) 341-344.

[3] Cavin J.F., Dartois V., Labarre C., Diviès C., Cloning of branched-chain amino acid biosynthesis genes and assays of $\alpha$-acetolactate synthase activities in Leuconostoc mesenteroides subsp. cremoris, Res. Microbiol. 150 (1999) 189-198.

[4] Cogain-Bousquet M., Garrigues C., Novak L., Lindley N. D., Loubière P., Rational development of a simple synthetic medium for the sustained growth of Lactococcus lactis, J. Appl. Bacteriol. 79 (1995) 108-116.

[5] Deguchi Y., Morishita T., Nutritional requirements in multiple auxotrophic lactic acid bacteria: genetic lesions affecting amino acid biosynthetic pathways in Lactococcus lactis, Enterococcus faecium, and Pediococcus acidilactici, Biosci. Biotechnol. Biochem. 56 (1992) 913-918.

[6] Desmazeaud M., L'état des connaissances en matière de nutrition des bactéries lactiques, Lait 63 (1983) 267-316.

[7] Devereux J., Haeberli P., Smithies O., A comprehensive set of sequence analysis programs for VAX, Nucl. Acids Res. 12 (1984) 387-395.

[8] Garault P., Letort C., Juillard V., Monnet V., Branched-chain amino acid biosynthesis is essential for Streptococcus thermophilus optimal growth in milk, Appl. Environ. Microbiol. 66 (2000) 5128-5133.

[9] Godon J.J., Chopin M.C., Ehrlich S.D., Branched-chain amino acid biosynthesis genes in Lactococcus lactis subsp. lactis, J. Bacteriol. 174 (1992) 6580-6589.

[10] Godon J.J., Delorme C., Bardowski J., Chopin M.C., Ehrlich S.D., Renault P., Gene inactivation in Lactococcus lactis: branched-chain amino acid biosynthesis, J. Bacteriol. 175 (1993) 4383-4390.

[11] Goupil-Feuillerat N., Cogain-Bousquet M. Godon J.J., Ehrlich S.D., Renault P., Dual role of $\alpha$-acetolactate decarboxylase in L. lactis subsp. lactis, J. Bacteriol. 179 (1997) 6285-6293.

[12] Huggins A.M., Sandine W.E., Differenciation of fast and slow milk coagulating isolates in strains of streptococci, J. Dairy Sci. 67 (1984) 1674-1679.

[13] Keilhauer C., Eggleling L., Sahn H., Isoleucine synthesis in Corynebacterium glutamicum molecular analysis of the $i l v B-i l v N-i l v C$ operon, J. Bacteriol. 175 (1993) 5595-5603. 
[14] Maguin E., Prévost H., Ehrlich S.D., Gruss A Efficient insertional mutagenesis in lactococci and other gram-positive bacteria, J. Bacteriol. 178 (1996) 931-935.

[15] Mierau I., Kunji E.R.S., Leenhouts K.J. Hellendorn M.A., Haandrikman A.J., Poolman B., Konings W.N., Venema G., Kok J., Multiple peptidase mutants of Lactococcus lactis are severely impaired in their ability to grow in milk, J. Bacteriol. 178 (1996) 2794-2803.

[16] Morishita T., Deguchi Y., Yajima M., Sakurai T. Yura T., Multiple nutritional requirements of lactobacilli: genetic lesions affecting amino acid biosynthetic pathways, J. Bacteriol. 148 (1981) 64-71.

[17] Neviani E., Giraffa G., Brizzi A., Carminati D., Amino acids requirements and peptidase activities of Streptococcus salivarius subsp. thermophilus, J. Appl. Bacteriol. 79 (1995) 302-307.

[18] Nilsson D., Kilstrup M., Cloning and expression of the Lactococcus lactis purDEK genes, required for growth in milk, Appl. Environ Microbiol. 64 (1998) 4321-4327.

[19] Poolman B., Konings W.N., Relation of growth of Streptococcus lactis and Streptococcus cremoris to amino acid transport, J. Bacteriol. 170 (1988) 700-707.

[20] Rallu F., Gruss A., Maguin E., Lactococcus lactis and stress, Antonie van Leeuwenhoek 70 (1996) 147-159.

[21] Renna M.C., Najimudin N., Winik L.R., Zahler S.A., Regulation of the Bacillus subtilis alsS, als $D$, and als $R$ genes involved in post-expo- nential-phase production of acetoin, J. Bacteriol. 175 (1993) 3863-3875.

[22] Shahbal S., Hemme D., Desmazeaud M., High cell wall-associated proteinase activity of some Streptococcus thermophilus strains (H-strains) correlated with a high acidification rate in milk, Lait 71 (1991) 351-357.

[23] Thomas T.D., Mills O.E., Proteolytic enzymes of starter bacteria, Neth. Milk Dairy J. 35 (1981) 255-273.

[24] Thomas T.D., Turner K.W., Preparation of skim milk to allow harvesting of starter cells from milk cultures, N. Z. J. Dairy Sci. Technol. 12 (1977) 15-21

[25] Tsau J.L., Guffanti A.A., Montville T.J., Conversion of pyruvate to acetoin helps to maintain $\mathrm{pH}$ homeostasis in Lactobacillus plantarum, Appl. Environ. Microbiol. 58 (1992) 891-894.

[26] von Wright A., Tynkkynen S., Souminen M., Cloning of a Streptococcus lactis subsp. lactis chromosomal fragment associated with the ability to grow in milk, Appl. Environ. Microbiol. 53 (1987) 1584-1588.

[27] Wang H., Yu W., Coolbear T., O'Sullivan D., McKay L., A deficiency in aspartate biosynthesis in Lactococcus lactis subsp. lactis C2 causes slow milk coagulation, Appl. Environ. Microbiol. 64 (1998) 1673-1679.

[28] Wek R.C., Hatfield G.W., Nucleotide sequence and in vivo expression of the $i l v Y$ and $i l v C$ genes in Escherichia coli K12, J. Biol. Chem. 261 (1986) 2441-2550. 